\title{
Motion structure in five-dot patterns as a determinant of perceptual grouping
}

\author{
ERIK BÖRJESSON and ULF AHLSTRÖM \\ Uppsala University, Uppsala, Sweden
}

\begin{abstract}
The effect of basic motion structures on perceptual grouping was studied with five-dot motion patterns. Four basic motion structures were identified in terms of proximal common and relative motion vectors. In a forced-choice situation, the observers had to decide to which of two pairs of dots a fifth critical dot seemed to belong. Thus, one of two possible three-dot units was chosen by the observers. The two possible three-dot units defined different motion structures, and the chosen motion structure was considered to have stronger grouping power than the alternative structure had. It was found that parallel common motions (perceived translation in the plane) had the strongest grouping power; these were followed by circular common motions (perceived rotation in the plane), concurrent relative motions (perceived translation in depth), and, finally, parallel relative motions (perceived rotation in depth). The results also suggested effects of proximity and orientation of axis of rotation. It is further argued that the relative grouping power of the motion structures could not solely be interpreted in terms of changes of directions and distances between the dots. Instead it is suggested that vector analysis is a fundamental perceptual activity and that basic motion structures determine grouping power.
\end{abstract}

Several researchers have used simple stimuli to study the limits of information for veridical event perception. Usually a restricted number of dots or other simple carriers of motion have been used. The main interest has generally been focused on the information inherent in the kinematic properties of perceptual units. It has usually been taken for granted that the moving elements have been perceived as grouped into perceptual units or in some other way related to each other, rather than perceived as separate, completely independent elements. For instance, all theorizing and stimulus construction in connection with perceptual vector analysis is founded on the assumption that individual dots are perceived in relation to each other. At its very core, the notion of perceptual vector analysis is founded on relations. Johansson's studies on vector analysis in a frontoparallel plane (1950), in depth (1964), and as applied to biological motion (1973) are examples of this foundation. Among others, Gogel (1974), Börjesson and von Hofsten $(1972,1973,1975)$ and Wallach, Becklen, and Nitzberg (1985) have used simple motion patterns under the tacit assumption that elements are grouped. Similarly, studies on minimal conditions for perceiving veridical structure from motion do assume that elements are grouped as perceptual units, rigid or nonrigid. However, it has occasionally been found that the formation of perceptual units does not occur as expected. Even in cases when the proximal pattern has been a projection of a rigid distal configuration, observers have sometimes failed to perceive a single perceptual unit. For

Correspondence should be addressed to E. Börjesson, Department of Psychology, Uppsala University, Box 1854, S-751 48 Uppsala, Sweden (e-mail: psyk@strix.udac.uu.se). instance, using four- and five-point displays, Braunstein, Hoffman, Shapiro, Andersen, and Bennett (1987) found that the subjects sometimes perceived the pattern as a triangle and a dot or as a triangle and a line. The same findings have been noted by Dosher, Landy, and Sperling (1989). According to them, a stimulus with a small number of dots can easily be divided into subgroups of two or three dots moving independently of each other.

Studies of perceptual vector analysis have described how common and relative motions are extracted. Studies of perceived structure from motion have suggested several constraints in order to explain correspondences from two to three dimensions. However, neither approach tells under what conditions the perceptual grouping appear in the first place. The general objective of the present study was to identify motion characteristics that promote formation of perceptual groupings.

The well-known Gestalt laws (Wertheimer, 1923) describe static conditions that promote formation of perceptual grouping. One law, that of common fate, identifies a motion characteristic that has a very great strength for creating perceptual grouping. The present problem was to find other candidates among motion structures that might have similar effects on perceptual grouping.

Working with small visual angles, Börjesson and von Hofsten (1975) have suggested a set of well-defined proximal motion patterns that comprise all possible rigid motions in space-that is, translation along and rotation around the three dimensions in space. In Figure 1, typical instances of these patterns are shown with two dots (A) and three dots (B).

The arrows show the motion tracks of the dots. In most of Börjesson and von Hofsten's (1975) experiments, the 


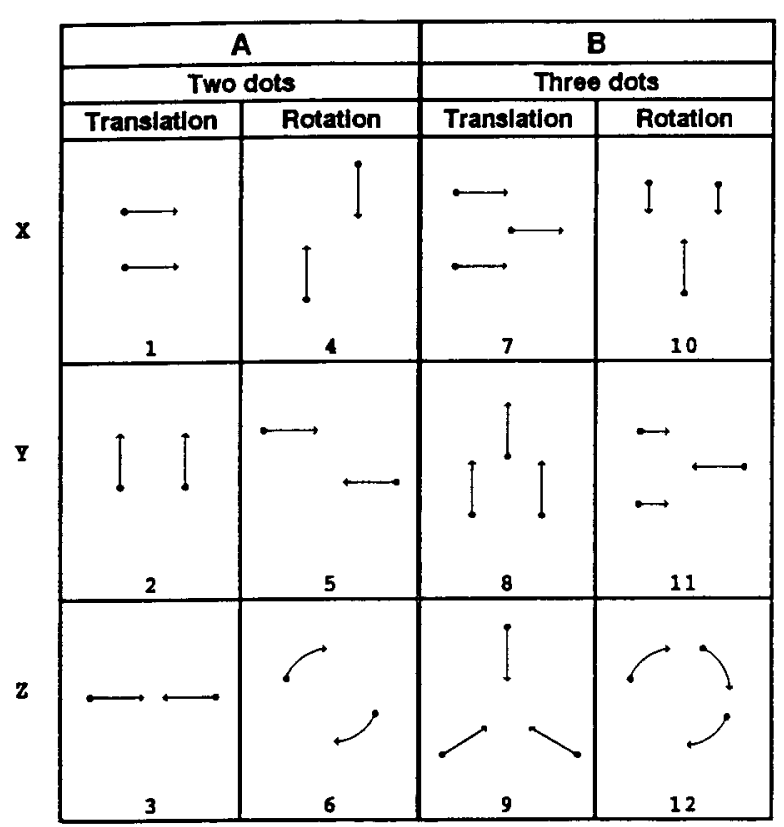

Figure 1. Motion structures of two (A) and three (B) dots for translation along and rotation around the three dimensions in space.

dots moved back and forth in their respective paths with constant velocity, appearing on an oscilloscope screen as small bright dots. Börjesson and von Hofsten $(1972,1975)$ classified the motion structures shown in Figure 1 in terms of their proximal kinematic characteristics rather than in terms of the type of motion in three-dimensional space that they represented. They first distinguished between common and relative motions. Common motion vectors are present when all dots move the same distance in the same direction, as is the case with Motion Structures 1, 2, 7, and 8 in Figure 1. Relative motions are present when there is a change of distance between the dots, as is the case with Motion Structures 3, 4, 5, 9, 10, and 11 in Figure 1 . Two types of relative motion were identified. First, so-called concurrent relative motions describe the case when the dots would meet if their motion toward each other would continue as in Motion Structures 3 and 9. Second, so-called parallel relative motions describe the case when the dots will never meet. This is the case with Motion Structures 4, 5, 10, and 11 in Figure 1. The remaining Motion Structures 6 and 12 in Figure 1 were classified by Börjesson and von Hofsten (1975) as relative motions, since the motion vectors of the dots are not the same with regard to direction. However, there is none of the change of distance between the dots that is a prerequisite for relative motions. Therefore, the circular motion structures will be considered as common motions in the present paper. To sum up, four motion structures will be studied with regard to grouping power. They will be referred to as basic motion structures, and they will have the following characteristics. All basic motion structures are composed of three dots. (1) For parallel common mo- tions (PCMs), the instantaneous motion vector has the same magnitude and direction for all three dots. PCMs correspond to the motions of the corners of a rigid triangle translating in a frontoparallel plane. (2) In circular common motions (CCMs), the instantaneous motion vector for each dot can be described relative to the line connecting the dot with the center of gravity for all three dots. The motion vector for each dot has a direction that is perpendicular to this line (rotated $90^{\circ}$ relative to the line clockwise or counterclockwise for all three dots) and a magnitude that is proportional to this line. The vector sum for the three dots equals zero. CCMs correspond to the motions of the corners of a rigid triangle rotating in a frontoparallel plane around the line of sight going through the center of gravity of the triangle. (3) In concurrent relative motions (CRMs), the instantaneous motion vectors of the three dots are directed toward (or away from) the center of gravity of the three dots. The magnitude of the motion vector for each dot is proportional to the distance between the dot and the center of gravity. The vector sum of the three dots equals zero. CRMs correspond to a polar projection of the three corners of a rigid triangle that translates in depth along the line of sight going through the center of gravity of the triangle. (4) In parallel relative motions (PRMs), the instantaneous motion vector for each dot can be described relative to a line in the frontoparallel plane going through the center of gravity of the three dots. The instantaneous motion vectors of the three dots are directed toward (or away from) this line. The magnitude of the motion vector for each dot is proportional to the distance between the dot and the line. As a consequence, all motion vectors are parallel and their sum equals zero. PRMs correspond to a parallel projection of the three corners of a rigid triangle rotating in depth around an axis on the triangle going through the center of gravity of the triangle.

Another model of proximal motions that is related to the present approach has been presented by Koenderink and van Doorn (e.g., 1975; Koenderink, 1986). They have proposed that the visual system is sensitive to the differential components of image deformations of a local surface patch in motion. These components are divergence, curl, and deformation. The basic motion structure of PCMs represents an absence of local image deformations, so that the differential components all are zero. The basic motion structure of CCMs is described by pure curl, and the basic motion structure of CRMs is described by pure divergence. The basic motion structure of PRMs, finally, is described by a combination of divergence and deformation.

\section{EXPERIMENT 1}

In Experiment 1, three motion structures were studied with regard to grouping power: PCMs, CRMs, and PRMs. PCMs were expected to have the strongest grouping power. The effect of proximity was also tested in Experiment 1 . Proximity is well known as a grouping fac- 
tor in static patterns. It also affects perceptual vector analysis in simple motion patterns with dots (Gogel, 1974). Gillam and Grant (e.g., 1984) have shown that proximity favors the grouping of moving lines.

The common dot technique. In Experiments 1-3, each stimulus pattern consisted of two simultaneously presented motion structures of three dots each, similar to those in Figure 1. This would yield six dots in all of the stimulus patterns. However, it would then be possible for the subjects to perceive two units of three dots each at the same time, and it would not be possible to decide which unit had the greater grouping power. Therefore, a forcedchoice situation was created in the following way. The motion structures were designed so that any two structures had one dot in common, the common dot; that is, one of the dots in one structure moved in exactly the same way as did one of the dots in the other structure. The common dots from the two constituting structures were superimposed in the stimulus patterns so that the pattern actually contained only five dots. Three instances (A-C) of this technique are shown in Figure 2. Each pattern consists of five dots. The three dots in each pattern that are denoted by the same symbols, circles, or squares belong to the same motion structure. The rightmost dot denoted by both a circle and a square belongs to both motion structures. This is the common dot. It can be perceived as a part of one of the structures but not of both structures at the same time. For instance, in Pattern A in Figure 2,

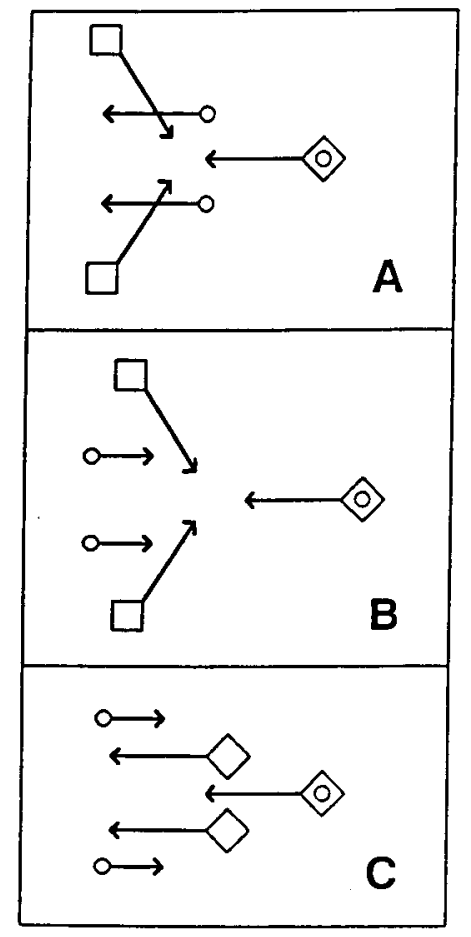

Figure 2. The common dot technique as applied to three motion patterns (A-C). Each pattern consists of two motion structures (squares or circles) with three dots. The rightmost dot, the common dot, belongs to both motion structures. one structure might evoke translation in depth and the other might evoke translation in a frontoparallel plane. The common dot must then be perceived to move either in depth or in the frontoparallel plane, depending on the structure to which it perceptually belongs. In this way, it was possible to study which three-dot motion structure would be perceived by the subjects and would thereby prove to have grouping power greater than that of the alternative structure.

\section{Method}

Apparatus. A PDP-11/10 computer was programmed to generate the stimulus patterns. The refresh rate is dependent on the load on the computer and cannot easily be decided. It was high enough to avoid flickering and to give the impression of smooth motions. The patterns were displayed by an oscilloscope (Tektronix, 604 Monitor). In front of the screen, a collimator lens was placed at a distance of $50 \mathrm{~cm}$. The lens gave approximately parallel light rays.

Stimuli. In all the experiments to be reported, conditions favoring grouping were provided, which means that the visual angles were not too great-generally, they were about $5^{\circ}$ of visual angle or less-and that the motions were not too swift or slow, about $1^{\circ}$ of visual angle per second or less. These levels were chosen from preliminary observations by the authors. No other specific constraints were put on the patterns, apart from characteristics needed to accomplish the objectives of the experiments. As has already been mentioned, the stimulus patterns consisted of five dots moving back and forth in their respective straight paths to complete a cycle in $5 \mathrm{sec}$. Three different basic motion structures, all defined by three dots, were used in Experiment 1: PCMs, CRMs, and PRMs. In each stimulus pattern, two of these structures were shown with one common dot. The distance between the dots was varied in three levels for each motion structure. Figure 3 shows how the patterns were constructed.

In Figure 3A, the simultaneous presentations of PCM and CRM are shown. The rightmost dot (a) is always the common dot. There are three pairs of dots $(b-c, d-e$, and $f-g)$ that each combine with dot a to define PCM. The three different pairs represent different distances between the dots. Furthermore, there are three pairs of dots $(h-i, j-k$, and $1-m)$ that each combine with dot a to define CRM. Each stimulus pattern consisted of dot a, one of the PCM pairs, and one of the CRM pairs. This makes nine different five-dot patterns. In the same way CRMs and PRMs are shown in Figure 3B, and PCMs and PRMs are shown in Figure 3C, always with the rightmost dot as the common dot. The combinations of dots in the 27 different stimulus patterns and the types of motion structure are explicated in Table 1.

Viewing conditions. Each subject sat about $21 \mathrm{~cm}$ in front of the collimator lens and looked binocularly through it at the dots that appeared on the screen. The position of the head was controlled by a chinrest. During the presentations, the room was darkened, and between each presentation, when the subjects gave their reports, the light was switched on.

Procedure. The subjects were shown three introductory test patterns with three dots only. The test patterns were instances of PCMs, CRMs, and PRMs. All subjects perceived these patterns as translation in a frontoparallel plane, translation in depth, and rotation in depth, respectively. The subjects were then told that they would be shown patterns with more dots and that they would identify perceptual units of the kind they had just been shown. No information was provided about how the stimulus patterns were constructed. The subjects were asked to report which three dots belonged together and also how this unit moved in space. Informal observations had shown that a shift sometimes occurred between different units. Therefore, the subjects were told to report only the threedot unit that appeared most stable in cases when the percept altered between different three-dot units. After that, five patterns, randomly 


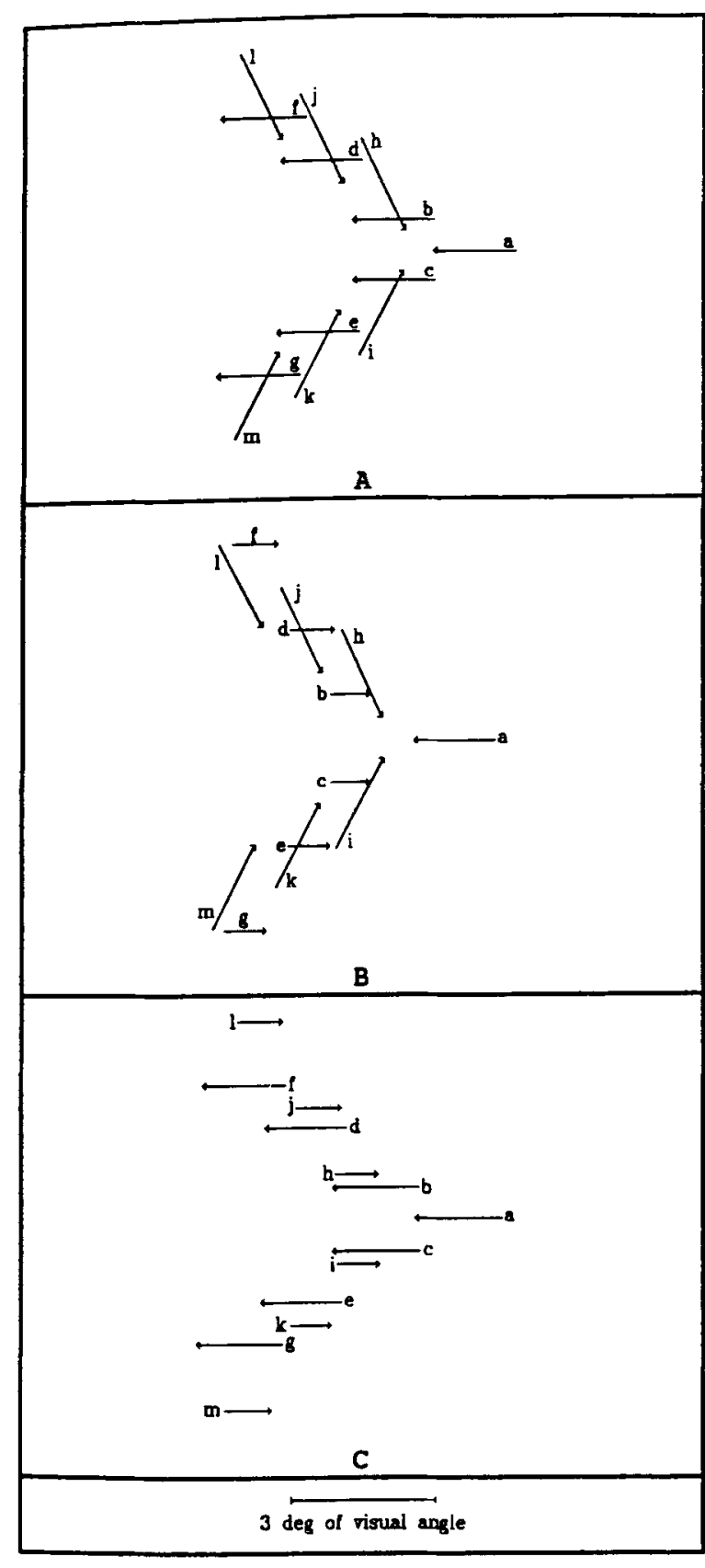

Figure 3. Stimulus patterns used in Experiment 1.

chosen from the stimulus patterns proper, were shown in order to ensure that the subjects had understood their task and could communicate which dots they perceived to be a unit and how that unit moved. No problems were found in these respects. Thereafter, the 27 stimulus patterns were shown once in randomized order. Each subject received a unique order. After each presentation, the subject reported the three dots and how they moved in space.

Subjects. Twelve undergraduate students of psychology participated in Experiment 1.

\section{Results and Discussion}

The subjects always chose three dots that made up a motion structure as defined above. In no case were three other dots chosen as a perceptual unit. Out of a total of 324 reports $(12 \times 27), 303$ supported the earlier findings by Börjesson and von Hofsten $(1972,1973,1975)$ that PCMs yield translation in a frontoparallel plane, CRMs yield translation in depth, and PRMs yield rotation in depth.

In Table 1, the two motion structures that constituted each pattern are shown in two three-dot combinations that refer to Figure 3. Within the parentheses immediately to the right of each dot combination is shown the number of subjects (out of 12) who perceived that particular dot combination as a unit. It is obvious that PCMs have the strongest grouping power. When this motion structure was present, it was preferred in $90.3 \%$ of the cases. When CRMs and PRMs were shown simultaneously, CRMs were preferred in $81.5 \%$ of the cases. From Experiment 1 , it is clear that PCMs have the strongest grouping power, that CRMs come next, and that PRMs have the weakest grouping power. The proximity of dots had also an effect, although it was much weaker. The proximity favors perceptual grouping. Considering only patterns with PCMs, we found that this motion structure was chosen in 69 cases (out of 72 summarized over 12 subjects and six patterns) when the dots of the PCMs were closest to each other. With medium proximity, PCMs were chosen in 64 cases, and with low proximity, PCMs were chosen in 62 cases. The corresponding frequencies for CRMs were 35, 33, and 27. Thus there is a decrease in frequency of preferences when the distances between the dots of the CRMs increases. Finally, preferences for PRMs decrease also with increasing distance between dots; these were 19, 12, and 3, respectively, for small, medium, and great distances between dots. Although the effects are small, they are consistent for all types of motion structures.

To conclude, motion structure and proximity of motion elements affect perceptual organization. Within the variations used in Experiment 1, motion structure has a much stronger effect than does proximity.

\section{EXPERIMENT 2}

In Experiment 1, PCMs, CRMs, and PRMs were studied with straight motion paths. The fourth basic motion structure, CCMs, consists of elements moving in circular motion paths. It is of special interest to investigate this motion structure, since it belongs to the group of common motions-that is, motion structures with constant distances between the constituting elements. Thus one objective of Experiment 2 was to study the grouping power of CCMs. It was predicted that because CCMs maintain a fixed distance between elements, CCMs would have stronger grouping power than would the relative motions. It was also predicted that PCMs would have greater grouping power than would CCMs. Another objective concerns procedure. In Experiment 1, the subjects were presented with different motions before the data collection proper began. There is some uncertainty as to how this might affect the subjects. Because of this, the subjects in Ex- 
Table 1

The Number of Preferences for Each of Two Motion Structures in the Stimulus Patterns in Experiment 1

\begin{tabular}{|c|c|c|c|c|c|c|c|c|c|c|c|}
\hline \multirow[b]{3}{*}{ Pattern } & \multirow[b]{3}{*}{ Figure } & \multirow[b]{3}{*}{ Dots } & \multicolumn{9}{|c|}{ Motion Structure } \\
\hline & & & \multicolumn{3}{|c|}{ PCMs } & \multicolumn{3}{|c|}{ CRMs } & \multicolumn{3}{|c|}{ PRMs } \\
\hline & & & Small & Medium & Large & Small & Medium & Large & Small & Medium & Large \\
\hline 1 & $2 \mathrm{~A}$ & abchi & $a b c(12)$ & & & $\operatorname{ahi}(0)$ & & & & & \\
\hline 2 & $2 \mathrm{~A}$ & abcjk & $a b c(11)$ & & & & $a j k(1)$ & & & & \\
\hline 3 & $2 \mathrm{~A}$ & abclm & abc(12) & & & & & $\operatorname{alm}(0)$ & & & \\
\hline 4 & $2 \mathrm{~A}$ & adehi & & $\operatorname{ade}(10)$ & & ahi(2) & & & & & \\
\hline 5 & $2 \mathrm{~A}$ & adejk & & $\operatorname{ade}(12)$ & & & $\operatorname{ajk}(0)$ & & & & \\
\hline 6 & $2 A$ & adelm & & $\operatorname{ade}(11)$ & & & & $\operatorname{alm}(1)$ & & & \\
\hline 7 & $2 \mathrm{~A}$ & afghi & & & afg $(10)$ & $\operatorname{ahi}(2)$ & & & & & \\
\hline 8 & $2 \mathrm{~A}$ & afgjk & & & $\operatorname{afg}(11)$ & & $\operatorname{ajk}(1)$ & & & & \\
\hline 9 & $2 \mathrm{~A}$ & afglm & & & $\operatorname{afg}(12)$ & & & $\operatorname{alm}(0)$ & & & \\
\hline 10 & $2 B$ & abchi & & & & ahi(10) & & & $a b c(2)$ & & \\
\hline 11 & $2 \mathrm{~B}$ & $a b c j k$ & & & & & $\operatorname{ajk}(9)$ & & $a b c(3)$ & & \\
\hline 12 & $2 \mathrm{~B}$ & abclm & & & & & & $\operatorname{alm}(6)$ & $a b c(6)$ & & \\
\hline 13 & $2 \mathrm{~B}$ & adehi & & & & $\operatorname{ahi}(9)$ & & & & ade(3) & \\
\hline 14 & $2 \mathrm{~B}$ & adejk & & & & & ajk(11) & & & $\operatorname{ade}(1)$ & \\
\hline 15 & 2B & adelm & & & & & & $\operatorname{alm}(10)$ & & $\operatorname{ade}(2)$ & \\
\hline 16 & $2 B$ & afghi & & & & ahi(12) & & & & & $\operatorname{afg}(0)$ \\
\hline 17 & 2B & afgjk & & & & & ajk $(11)$ & & & & afg(1) \\
\hline 18 & 2B & afglm & & & & & & $\operatorname{alm}(10)$ & & & $\operatorname{afg}(2)$ \\
\hline 19 & $2 \mathrm{C}$ & abchi & $\operatorname{abc}(11)$ & & & & & & ahi(1) & & \\
\hline 20 & $2 C$ & abcjk & $a b c(11)$ & & & & & & & ajk(1) & \\
\hline 21 & $2 \mathrm{C}$ & abclm & $a b c(12)$ & & & & & & & & $\operatorname{alm}(0)$ \\
\hline 22 & $2 \mathrm{C}$ & adehi & & $\operatorname{ade}(8)$ & & & & & $\operatorname{ahi(4)}$ & & \\
\hline 23 & $2 C$ & adejk & & $\operatorname{ade}(11)$ & & & & & & $\operatorname{ajk}(1)$ & \\
\hline 24 & $2 \mathrm{C}$ & adelm & & $\operatorname{ade}(12)$ & & & & & & & $\operatorname{alm}(0)$ \\
\hline 25 & $2 C$ & afghl & & & $\operatorname{afg}(9)$ & & & & ahi(3) & & \\
\hline 26 & $2 \mathrm{C}$ & afgjk & & & $\operatorname{afg}(8)$ & & & & & ajk(4) & \\
\hline 27 & $2 C$ & afglm & & & $\operatorname{afg}(12)$ & & & & & & $\operatorname{alm}(0)$ \\
\hline$\Sigma$ & & & 69 & 64 & 62 & 35 & 33 & 27 & 19 & 12 & 3 \\
\hline
\end{tabular}

periment 2 were not exposed to any motions before the experiment. If great differences in the relative frequencies of different percepts should be found between the two experiments, the effect of procedure would have to be considered.

\section{Method}

The common dot technique used in Experiment 1 was also used in Experiment 2.

Apparatus. The stimulus patterns were programmed in Z-BASIC and generated on a Macintosh II computer screen. The spatial resolution of the screen was $76 \mathrm{dpi}$, and the displays were presented with a refresh rate of $67 \mathrm{~Hz}$. To minimize cues of two-dimensionality, a collimator lens was placed between the screen and the subject at a distance of $50 \mathrm{~cm}$ from the screen.

Stimuli. The stimulus patterns consisted of five dots moving back and forth in their respective paths. The motion in each direction lasted for $3 \mathrm{sec}$. With a pause at each end point, a full cycle was completed in $8 \mathrm{sec}$. Four motion structures were used in Experiment 2, PCMs, CCMs, CRMs, and PCMs/PRMs. PCMs/PRMs were an algebraic sum of PCMs and PRMs. Each motion structure consisted of three dots. One of these dots, the common dot, had the same real motion path in all motion strucrures. Since CCMs were one of the structures in Experiment 2, the common dot had to have a circular motion path. Since they consist of straight motion paths, PRMs could not share a common dot with CCMs unless PRMs were appropriately combined with some other motion component. Through addition of the PCMs to the PRMs it was possible to get a combined motion structure PCMs/PRMs that had two circular motion paths, one of which was identical with that of the common dot in Experiment 2 (a slash between two basic motion structures will be used to denote an algebraic sum between these structures). The PCMs/PRMs replaced PRMs under the assumption that the addition of PCMs, in this case, would not substantially increase the grouping power of the structure. This assumption was founded on informal observations before the experiment.

Of the four motion structures mentioned above, PCMs, CCMs, CRMs, and PCMs/PRMs, for each stimulus pattern, two were chosen and superimposed so that the common dots coincided. All possible pairs of four motion structures made six stimulus patterns. These are shown in Figure 4.

In Pattern A in Figure 4, PCMs and CCMs are compared. PCMs are represented by the parallel common motion paths (down left) of three dots-that is, of the two dots shown as squares and the common dot shown as a circle. The equilateral triangle constituted by these dots had sides covering $3.5^{\circ}$ of visual angle. The CCMs are represented by the motions of the two dots shown as triangles and the common dot. These dots constitute an imaginary equilateral triangle, the sides of which covered $3.5^{\circ}$ of visual angle. This triangle rotated $30^{\circ}$ around the line of sight.

In Pattern B in Figure 4, PCMs and CRMs are compared. The PCMs are the same as in Pattern A. The motion of the two dots shown as triangles and the common dot represent CRMs. These dots define an imaginary triangle that increased and decreased in size. The imaginary triangle's vertical extension varied between $2.5^{\circ}$ and $3.5^{\circ}$ of visual angle, respectively. The horizontal extension of the imaginary triangle varied between $2.5^{\circ}$ and $4.0^{\circ}$ of visual angle. It should be noted that whereas the dots in most cases moved with constant velocity in their respective paths, this was not the 


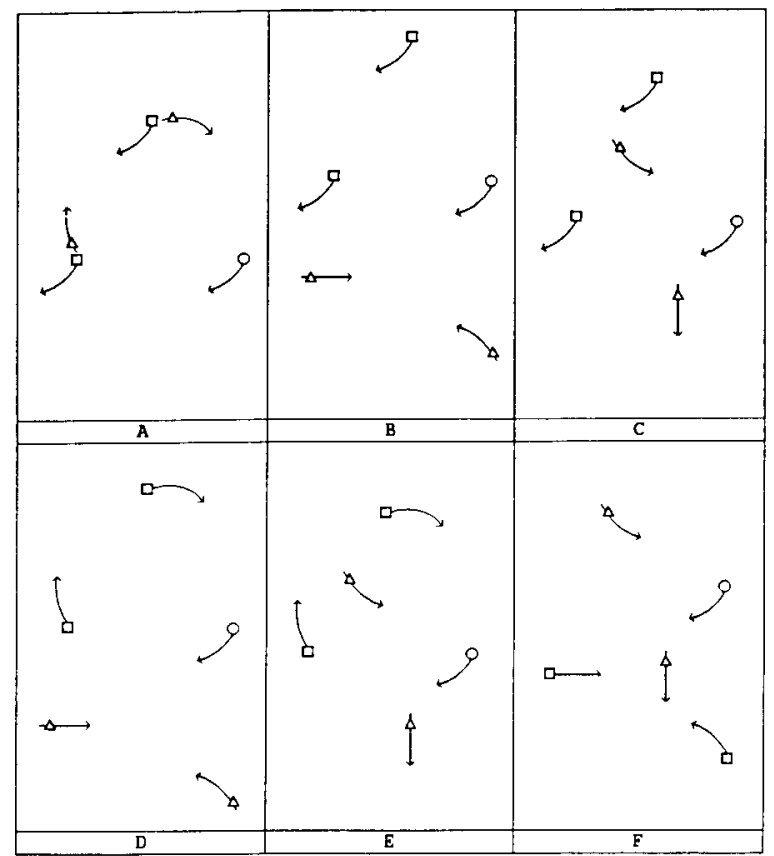

Figure 4. Stimulus patterns used in Experiment 2.

case with the leftmost horizontally moving dot of the CRMs. This dot slightly changed its velocity to match the change of direction of the two rightmost dots (with the effect of keeping the shape of the imaginary CRM triangle constant). This means that the velocity of the left dot always had the same magnitude as that of the horizontal component of the rightmost dots. It was impossible to see that there was a deviation from constant velocity. What is important is that the CRM triangle maintained its shape.

In Pattern $\mathrm{C}$ in Figure 4, PCMs and PCMs/PRMs are compared. The PCMs are the same as in Pattern A. The motion of the two dots shown as triangles and the common dot represent the motion structure for PCMs/PRMs. These dots define an imaginary triangle, the motion of which constitutes an algebraic sum of horizontal PRMs and vertical PCMs. As a consequence, the lowest dot did not move with constant velocity but changed its velocity in accordance with the vertical component of the other two PCM/PRM dots. Again, the change of velocity was not possible to see; the important thing is to keep the vertical distances of the PCM/PRM triangle constant. The triangle's vertical extension was $3.0^{\circ}$ of visual angle, the vertical displacement of the triangle was $0.6^{\circ}$ of visual angle, and the horizontal extension varied between $1.9^{\circ}$ and $2.4^{\circ}$ of visual angle.

In Pattern D in Figure 4, CCMs and CRMs are combined. The CCMs shown by the two squares and the common dot were the same as in Pattern A. The CRMs shown by the two triangles and the common dot were the same as in Pattern B.

In Pattern $E$ in Figure 4, CCMs and PCMs/PRMs are combined. The CCMs shown as two squares and the common dot were the same as in Pattern A. The vector structure for PCMs/PRMs, shown by the two triangles and the common dot, were the same as in Pattern E.

Finally, in Pattern F in Figure 4, CRMs and PCMs/PRMs are compared. The CRMs shown as two squares and the common dot were the same as in Pattern B. The vector structure for PCMs/PRMs shown by the triangles and the common dot was the same as in Pattern E.

Viewing conditions. The viewing conditions were the same as in Experiment 1.
Procedure. Before the experiment, the subjects were told that they were going to see moving dots on the computer screen, and that these dots could be perceived to move in different ways in space. The motion could be a translatory motion in the frontoparallel plane, a translatory motion in depth, a rotation in depth, or a circular motion around the line of sight. These alternatives were described in words and with hand gestures. The subjects were not presented with different patterns before the experiment, as had been done in Experiment 1 . They were told that their task was to verbally report the three dots in the five-dot motion pattern that they perceived as a distinct perceptual unit and also to report how this unit moved in space. In cases where there was a shift of perceptual unit, the subjects were asked to report just one unit, the most stable unit. Finally, the subjects were asked not to fixate a particular dot in the stimulus pattern during their inspection. After this instruction, the different stimulus patterns were presented. The room was darkened during each presentation and the subjects looked binocularly through the collimator lens at the moving dots on the computer screen until they were ready to give their reports. When the subjects reported their percepts, the room was lighted and the reports were written down. This procedure was repeated until all six patterns had been presented twice to each subject. For each subject, a different random order of the 12 stimulus presentations was prepared.

Subjects. Nineteen undergraduate psychology students participated in Experiment 2. None of them had participated in Experiment 1 .

\section{Results and Discussion}

The percepts reported by the subjects were classified into five different response categories. (1) Perceived translation in the frontoparallel plane (trans $p$ ). This percept indicates that the PCMs were extracted. (2) Perceived rotation around the line of sight (rot $z$ ). This percept indicates that the CCMs were extracted. (3) Perceived translation in depth (trans $d$ ). This percept indicates that the CRMs were extracted from the stimulus pattern. (4) Perceived rotation in depth ( $r o t d)$, which indicates that the PRMs were extracted from the stimulus pattern. (5) Other answers, indicating other percepts given by the subjects that could not be recorded in the four previous categories. In Table 2, the frequencies summarized over subjects and replications $(19 \times 2)$ of the different response categories for each stimulus pattern are shown.

The reports given by the subjects for Patterns A-F were expected to be classified in one of two different response

Table 2

The Frequencies of Different Response Categories for Each Stimulus Pattern in Experiment 2

\begin{tabular}{cccccc}
\hline & \multicolumn{5}{c}{ Response Category } \\
\cline { 2 - 6 } Pattern & trans $p$ & rot $z$ & trans $d$ & rot $d$ & $\begin{array}{c}\text { Other } \\
\text { Answers }\end{array}$ \\
\hline A & 29 & 9 & & & \\
B & 36 & & & 3 & 1 \\
C & 34 & & & & 4 \\
D & & 33 & 1 & 1 & 10 \\
E & & 27 & & 6 & 4 \\
F & & & 28 & 10 & 21 \\
E & 99 & 69 & 29 & 10
\end{tabular}

Note-trans $p$, perceived translation in the frontoparallel plane; rot $z$, perceived rotation around the line of sight; trans $d$, perceived translation in depth; rot $d$, perceived rotation in depth; other answers, other percepts that did not belong to the other four categories. 
categories for each pattern, depending on which motion structures were present in the stimulus pattern. For Pattern $\mathrm{A}$, the two alternatives were trans $p$ and rot $z$; for Pattern B, trans $p$ and trans $d$; for Pattern $\mathrm{C}$, trans $p$ and rot $d$; for Pattern D, rot $z$ and trans $d$; for Pattern $\mathrm{E}$, rot $z$ and rot $d$; and for Pattern F, trans $d$ and rot $d$. Patterns A-C in Table 2 show that PCMs have a stronger perceptual grouping effect than do CCMs (A), CRMs (B), and PCMs/PRMs (C). Patterns D and E in Table 2 show that CCMs have a stronger perceptual grouping effect than do CRMs (D) and PRMs (E). Finally, Pattern F in Table 2 shows that CRMs have a stronger perceptual grouping effect than PCMs/PRMs have. These results support the assumption that the addition of PCMs to PRMs did not substantially increase the grouping power, since PCMs/PRMs had the weakest grouping power in the experiment.

The results from Experiment 2 are similar to the results from Experiment 1 with regard to the strength of perceptual grouping effect from PCMs, CRMs, and PRMs. The experimental hypothesis that CCMs have a stronger grouping power than do relative motions was confirmed in Experiment 2.

The agreement between the results from Experiments 1 and 2 suggests that the difference in procedure did not affect the results.

In summary, it can be concluded that PCMs provide the strongest grouping power, followed by CCMs. CRMs come in the third place, followed by the motions that yield the weakest grouping power, PRMs.

\section{EXPERIMENT 3}

Experiments 1 and 2 have shown that the basic motion structures can be ordered in terms of their power to organize elements into perceptual units. The next question concerns possible factors that determine that order. There are at least two hypotheses to consider in this respect. One of them simply states that the basic motion structure as such determines grouping power. The other hypothesis, however, states that the changes in proximal directions and distances between dots will determine grouping power. If there are no changes, the grouping power will be great. If there are changes in both directions and distances between the dots, the grouping power will be small. Considering basic motion structures in terms of these changes, PCMs, with the greatest grouping power, have no changes in any respect. CCMs and CRMs, with intermediate grouping power, have changes in one respect; CCMs change directions between dots and CRMs change distances between dots. Finally, PRMs, which have the weakest grouping power, change both directions and distances between dots. Thus, it would be possible to explain the order of grouping power among the basic motion structures in terms of proximal changes of directions and distances between dots.

The aim of Experiment 3 was to test whether the order of grouping power could be accounted for in terms of proximal changes of directions and distances between dots or whether the basic motion structure as such determined that order.

\section{Method}

The four different basic motion structures have different combinations of changes of directions and distances between dots that end up in a confounding of basic motion structures and changes of directions and distances. The results obtained with basic motion structures in Experiments 1 and 2 could be accounted for by both interpretations. In order to resolve this situation, it was necessary to create a pattern the grouping power of which would depend on whether it was the motion structure as such or changes in directions and distances that determined grouping power. Such a pattern was created according to the following presupposition. If two basic motion structures are combined into a motion pattern, the grouping power of that pattern will not be weaker than the grouping power of the weakest of the constituting motion structures. Consider the motion pattern in Figure 5 , in which a combination of CCMs and CRMs (CCMs/CRMs) is shown.

The momentarily initial motion vectors are shown in terms of CCMs and CRMs in Figure 5A. The resulting motion paths are shown in Figure 5B. This motion can be thought of as a triangle rotating around the line of sight and translating in depth. The dotted lines in Figure 5B show the size and orientation of the imaginary triangle at its turning points. The motion pattern shown in Figure 5 is a combination of CCMs and CRMs that have changes of directions and distances between dots. This means that the pattern can be used to decide whether it is basic motion structure as such that determines grouping power or the changes in the directions and distances between dots that is the important determinant. If basic motion structure as such is the critical determinant, CCMs/CRMs would have greater grouping power than PRMs, according to the results from Experiments 1 and 2. If, on the other hand, changes of directions and distances between dots determine grouping power, CCMs/CRMs should have about the same grouping power as PRMs have.

Apparatus. This was the same as in Experiment 2.

Stimuli. The stimulus patterns consisted of five dots moving back and forth in their respective paths. The motion in each direction lasted for $3.3 \mathrm{sec}$. With a pause at each end point, a full cycle was completed in $8.6 \mathrm{sec}$. Three motion structures were used in Experiment 3: (1) CCMs/CRMs, (2) PCMs, and (3) PRMs. All structures consisted of three dots moving back and forth with constant velocity in straight motion paths. One of these dots, the common dot, had the same real motion path in all motion structures. The common dot technique from Experiments 1 and 2 was used in Ex-

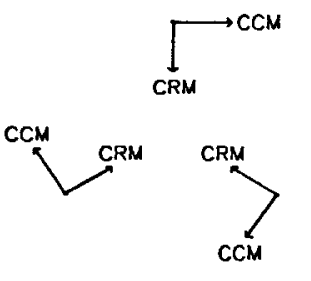

A

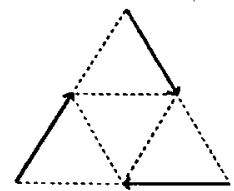

B
Figure 5. The combination of the basic motion structures, circular common motions (CCMs) and concurrent relative motions (CRMs). The momentarily initial velocity vectors are shown in $5 \mathrm{~A}$. The motion paths resulting from these vectors are shown as solid arrows in 5B. The dotted lines show the end positions of the triangle. 


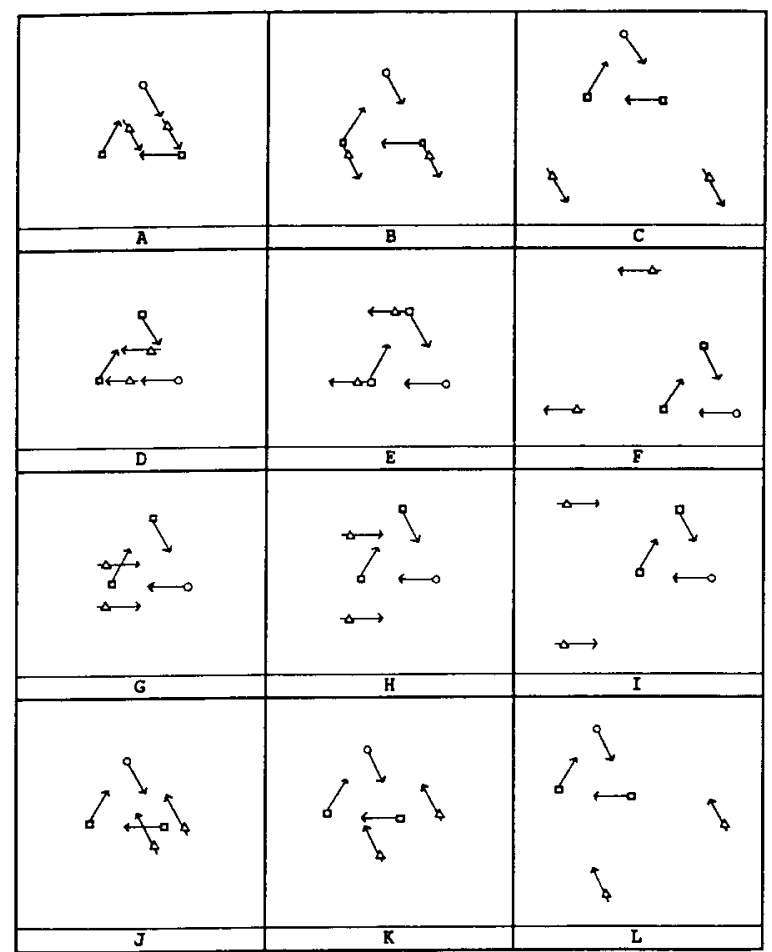

Figure 6. Stimulus patterns used in Experiment 3.

periment 3. The stimulus patterns used in Experiment 3 are presented in Figure 6.

The combination of CCMs/CRMs was present in every stimulus pattern, represented by the straight motion paths of the three dots constituting an imaginary equilateral triangle. In Figure 6, these dots are shown as two squares and a circle. The dot shown as a circle is the common dot. The CCMs/CRMs were compared with PCMs and with PRMs. In Patterns A-F, CCMs/CRMs were compared with PCMs. The PCMs are constituted by the two triangles and the circle. Thus the circle is a part of both the CCMs/CRMs and the PCMs. Either the orientations of the PCM vectors were oblique vertical, up/left, and down/right (Patterns A-C), or they were horizontal (Patterns D-F). The size of the imaginary equilateral triangle defined by the squares and the circle was always the same for all six patterns; the sides covered $3.5^{\circ}$ of visual angle when the triangle had its largest extension, and $1.8^{\circ}$ of visual angle at its smallest extension. In Figure 6, Patterns $\mathrm{A}, \mathrm{B}$, and $\mathrm{C}$ have oblique vertical orientations (up/left, down/right) of the PCM vectors. Together with the common dot, the two additional dots shown as triangles in Figure 6 defined an imaginary equilateral triangle. The size of this triangle varied in three steps, with the sides $1.8^{\circ}, 3.6^{\circ}$, and $7.0^{\circ}$ of visual angle for Patterns $\mathrm{A}, \mathrm{B}$, and $\mathrm{C}$, respectively. Patterns D, E, and F in Figure 6 have horizontal orientation of the PCM vectors. In these patterns too, the two additional dots together with the common dot defined an imaginary equilateral triangle. The size of this imaginary triangle varied in three steps, with the sides $1.8^{\circ}, 3.6^{\circ}$, and $7.0^{\circ}$ of visual angle, respectively.

In Patterns G-L in Figure 6, CCMs/CRMs were compared with PRMs. The PRMs are constituted by the two triangles and the circle. Thus the circle is a part of both the CCMs/CRMs and the PRMs. The size of the imaginary equilateral triangle, shown by two squares and the circle, was the same as for Patterns A-F, and the sides of the imaginary triangle varied with the same degrees of visual an- gle. The two additional dots, shown as triangles, together with the common dot, constitute an imaginary triangle that changes in accordance with PRMs. In Patterns G, H, and I, this imaginary triangle has horizontal orientations of the PRMs. The triangle's vertical extension was $1.8^{\circ}, 3.5^{\circ}$, and $6.0^{\circ}$ of visual angle, respectively, for Patterns G, H, and $\mathrm{I}$. The horizontal extension of the triangle varies between $0.4^{\circ}$ and $3.9^{\circ}$ of visual angle for Pattern G, $0.8^{\circ}$ and $4.3^{\circ}$ for Pattern $\mathrm{H}$, and finally, $3.4^{\circ}$ and $7.0^{\circ}$ of visual angle for Pattern I. Patterns J, K, and L have oblique vertical orientations (up/left, down/right) of the PRM vectors. The imaginary triangle, shown by triangles and a circle, that carries the PRMs is identical in size to those in Patterns G-I, but rotated $120^{\circ}$ counterclockwise around the line of sight.

Viewing conditions. These were the same as in Experiment 1.

Procedure. Before the experiment, the subjects were told that they were going to see moving dots on the computer screen, and that these dots could constitute different gestalts that moved in different paths in a three-dimensional space. The motion could be a translatory motion in depth with a simultaneous rotation around the line of sight, a translatory motion in the frontoparallel plane, or a rotation in depth. In three preliminary trials, the three different motion structures that appeared in the stimulus patterns used in Experiment 3-that is, CCMs/CRMs, PCMs, and PRMs-were presented in random order to the subjects. The subjects were asked to verbally describe the perceived motion of the three-dot patterns. After this, they were told that only two of the three possible gestalts would simultaneously appear in the stimulus patterns to be shown. The subjects were also told that the task was to report the gestalt they perceived as most distinct in the stimulus pattern. Finally, the subjects were asked not to fixate a particular dot in the stimulus pattern but to let the gaze move freely over the pattern. After this instruction, the different stimulus patterns were presented. During each presentation, the room was darkened and the subjects looked at the pattern until they were ready to give their reports. Then the room was lighted and the reports were written down. This procedure was repeated until the 12 patterns had been presented twice to each subject. For each subject, a different random order of the 24 stimulus presentations was prepared.

Subjects. Thirteen undergraduate psychology students participated in Experiment 3. None had participated in Experiment 1 or 2.

\section{Results and Discussion}

The subjects were presented with the three motion structures that appeared in the 12 stimulus patterns shown in Figure 4. All subjects perceived CCMs/CRMs as a rotation around the line of sight and a simultaneous translation in depth, PCMs as a translation in the frontoparallel plane, and PRMs as rotation in depth. The subjects' reports in response to the experimental patterns were classified into four different categories: (1) Perceived rotation around the line of sight and a simultaneous translation in depth (rot $z /$ trans $d$ ); this percept indicates that the CCMs/CRMs were perceived as a perceptual unit. (2) Perceived translation in the frontoparallel plane (trans $p$ ); this percept indicates that the PCMs were perceived as a perceptual unit. (3) Perceived rotation in depth (rot d); this percept indicates that the PRMs were perceived as a perceptual unit. (4) Other answers, indicating other percepts given by the subjects that could not be classified in the three previous categories.

The purpose of Experiment 3 was to examine the strength of perceptual grouping from different motion constellations. Table 3 shows the frequencies of different 
Table 3

The Frequencies of Different Response Categories for Each Stimulus Pattern in Experiment 3

\begin{tabular}{|c|c|c|c|c|c|c|c|}
\hline \multirow{2}{*}{\multicolumn{2}{|c|}{ CCMs/CRMs }} & \multirow{3}{*}{$\begin{array}{c}\text { Distance } \\
\text { Between } \\
\text { Dots }\end{array}$} & \multirow{3}{*}{$\begin{array}{l}\text { Orientation } \\
\text { of Motion } \\
\text { Vectors }\end{array}$} & \multicolumn{4}{|c|}{ Response Category } \\
\hline & & & & \multirow{2}{*}{$\begin{array}{l}\text { rot } z / \\
\text { trans d }\end{array}$} & \multirow[b]{2}{*}{ trans $p$} & \multirow[b]{2}{*}{ rot $d$} & \multirow{2}{*}{$\begin{array}{c}\text { Other } \\
\text { Answers }\end{array}$} \\
\hline PCMs & PRMs & & & & & & \\
\hline A & & small & oblique & 1 & 25 & & \\
\hline B & & medium & " & 3 & 22 & & 1 \\
\hline $\mathrm{C}$ & & large & " & 1 & 25 & & \\
\hline D & & small & horizontal & & 26 & & \\
\hline $\mathrm{E}$ & & medium & $"$ & 1 & 25 & & \\
\hline F & & large & $"$ & 2 & 24 & & \\
\hline & G & small & $"$ & 14 & & 10 & 2 \\
\hline & $\mathbf{H}$ & medium & $"$ & 17 & & 9 & \\
\hline & I & large & $"$ & 19 & & 6 & 1 \\
\hline & $\mathbf{J}$ & small & oblique & 21 & & 5 & \\
\hline & $\mathbf{K}$ & medium & " & 18 & & 7 & 1 \\
\hline & $\mathbf{L}$ & large & $"$ & 22 & & 3 & 1 \\
\hline$\Sigma$ & & & & 119 & 147 & 40 & 6 \\
\hline
\end{tabular}

Note-CCMs, circular common motions; CRMs, concurrent relative motions; PCMs, parallel common motions; PRMs, parallel relative motions; rot $z$, perceived rotation around the line of sight; trans $d$, perceived translation in depth; trans $p$, perceived translation in the frontoparallel plane; rot $d$, perceived rotation in depth; other answers, other percepts that did not belong to the other four categories.

kinds of percepts for each stimulus pattern used in $\mathrm{Ex}$ periment 3.

The reports given by the subjects from Stimulus Patterns A-F were expected to be classified in one of two different response categories-that is, rot $z /$ trans $d$ if the $\mathrm{CCMs} / \mathrm{CRMs}$ were extracted from the stimulus pattern, or trans $p$ if the PCMs were extracted. The reports from stimulus Patterns G-L were expected to be classified in one of two response categories-that is, rot z/trans $d$ if the CCMs/CRMs were extracted and rot $d$ if the PRMs were extracted. Patterns A-F in Table 3 show that PCMs have a stronger perceptual grouping power than CCMs/ CRMs have. The most interesting comparison concerns the CCMs/CRMs and the PRMs that both have changes of directions and distances between the dots. As can be seen in Table 3, CCMs/CRMs are preferred to PRMs in all six patterns (Patterns G-L). It can be concluded that the occurrence of changes of directions and distances between the dots cannot account for the different grouping powers of CCMs/CRMs and PRMs. The alternative hypothesis states that there are inherent differences between basic motion structures in this respect.

To get a variety of patterns, the distance between dots and orientation of the PCMs and the PRMs were varied in Experiment 3. Since the PCMs were almost always preferred to the CCMs/CRMs, effects of size and orientation could not be studied with PCMs. With PRMs, however, it was found that orientation had a slight effect. Over subjects, horizontal PRMs were preferred 25 times (out of 78), whereas oblique PRMs were preferred only 15 times. Although this difference is not significant, it is supported by other results attributed to the oblique effect (Appelle, 1972). Distance between dots in the PRM patterns had also a slight yet not significant effect. With small distances between the dots, the total sum of preferences of
PRMs were 15 (out of 52). The corresponding values for medium and large distances between dots were 16 and 8 , respectively. It seems, in accordance with the results from Experiment 1 and the Gestaltist law of proximity that small distances between moving dots favor perceptual grouping.

\section{DISCUSSION}

The general aim of this study was to identify motion characteristics of simple motion patterns that affect perceptual grouping power. It can be concluded that the type of basic motion structure is a strong determinant of perceptual grouping. Among basic motion structures, PCMs have the strongest grouping effect, followed by CCMs and CRMs. PRMs have the weakest grouping effect. It should be noted that in the present paper basic motion structure has explicitly been defined in terms of three moving elements and that consequently only the unit formation of three elements has been studied. Therefore, it is not possible to generalize from the results and conclusions to patterns with less or more than three elements. Additional elements provide new information and it is probable, to say the least, that the visual system applies other constraints and performs other operations when more elements are added.

A proximal analysis in terms of the occurrence of changes of directions and distances between moving elements has been considered. For instance, PCMs have no such changes, whereas PRMs have both types of changes. Even if this factor might be of importance, it does not tell the whole story, since, as was shown in Experiment 3, $\mathrm{CCMs} / \mathrm{CRMs}$ were in all instances preferred to PRMs. Apparently, the CCMs/CRMs were analyzed into CCMs and CRMs, and, as a consequence, these motions were 
more easily perceived as a group than were PRMs. This suggests that vector analysis is a very fundamental perceptual activity and that the type of the basic motion structure determines grouping power rather than proximal changes of directions and distances between the dots.

In addition to the type of basic motion structure, the proximity of the dots was studied. Experiments 1 and 3 showed consistent effects of proximity. With the patterns used, proximity favored perceptual grouping, although this effect was not as strong as that of motion structure.

Finally, a relatively weak, nonsignificant effect of orientation was found in Experiment 3. It seems that horizontal PRMs are more easily grouped than oblique PRMs, which supports informal observations of other patterns we have made and is in accordance with the "oblique" effect (Appelle, 1972).

The size of motion vectors might affect grouping power. It seems reasonable to assume that an increase, at least to a limit, favors perceptual grouping. This implies a problem in the comparison of different basic motion structures, since different parameters can be of importance for the different structures. Consider, for instance, CCMs, with which a given size of the velocity vectors implies different angles of rotation around the line of sight for different sizes of the imaginary triangle. Small distances between the dots give a great angle of rotation, and great distances give a smaller angle. It is not clear whether the angle of rotation or the absolute size of the velocity vectors is the most effective factor. In a similar way, a given size of velocity vectors has different effects on the relative changes of size and shape in CRMs and PRMs when the initial distances between the moving elements are changed. Large imaginary triangles are of course undergoing a relatively smaller change of size or shape than are smaller triangles, given a constant absolute size of velocity vectors. In the present experiments, the initial sizes of the imaginary triangles were kept approximately constant (except in cases when they were deliberately varied), as were the sizes of the velocity vectors. The common dot technique, for instance, had the effect that certain aspects of velocity vectors had to be constant.

In Experiment 3, we used a combination of CCMs and CRMs. We took it for granted that the occurrence of two basic motion structures in one pattern did not add or subtract anything to the grouping power. In any case, the superiority of CCMs/CRMs relative to PRMs should not be attributed to the fact that two basic motion structures were present in the same pattern. In Experiment 2, PCMs and PRMs were combined in a single pattern, PCMs/ PRMs, and there was no evidence that the combination as such increased grouping power. On the contrary, PCMs/PRMs had the weakest grouping power in Experiment 2. The effect of combinations as such of basic motion structures on grouping power is, however, unclear.

As has been suggested above, vector analysis is a fundamental perceptual activity, and the basic motion struc- tures have inherent grouping powers. If this is true, other operations than those used in this study should yield similar results. Lappin, Norman, and Mowafy (1991) studied the detectability of coherence in rapidly changing optical patterns. Following Koenderink and van Doorn (e.g., 1975; Koenderink, 1986), they studied the visual system's sensitivity to translations, rotations, expansions, pure shear, and combinations of these. Lappin et al. concluded that the visual system is sensitive to the different transformations. This conclusion gives indirect support to the view that dots moving according to specific motion structures are grouped and perceived as units. However, Lappin et al. also concluded that the transformations that they studied could not be considered as visually independent or as "visual primitives." Their conclusion was based mainly on the finding that the visual system did not effectively analyze combined transformations. Whereas rotation and expansion when combined were effectively analyzed and found to be independent, other combinations were less accurately analyzed, especially those including shear. These results are related to the present findings. First, Experiment 3 has shown that vector analysis into CCMs and CRMs (rotation and expansion) is easily made by the visual system. Second, all the experiments in the present study have shown that PRMs, the only basic motion structure with a component of shear, had the least grouping power. It seems that motions resulting in deformation of shape, regardless of change of area, are somewhat more difficult to analyze.

\section{REFERENCES}

Appelle, S. (1972). Perception and discrimination as a function of stimulus orientation: The "oblique effect" in man and animals. Psychological Bulletin, 78, 266-278.

Braunstein, M. L., Hoffman, D. D., Shapiro, L. R., Andersen, G. J., \& BENNETT, B. M. (1987). Minimum points and views for the recovery of three-dimensional structure. Journal of Experimental Psychology: Human Perception \& Performance, 13, 335-343.

BörJesson, E., \& Hofsten, C. von. (1972). Spatial determinants of depth perception in two-dot motion patterns. Perception \& Psychophysics, 11, 263-268.

Böruesson, E., \& Hofsten, C. von. (1973). Visual perception of motion in depth: Application of a vector model to three-dot motion patterns. Perception \& Psychophysics, 13(2), 169-179.

BörJesson, E., \& Hofsten, C. von. (1975). A vector model for perceived object rotation and translation in space. Psychological Research, 38, 209-230.

Dosher, B. A., Landy, M. S., \& SPerling, G. (1989). Ratings of kinetic depth in multidot displays. Joumal of Experimental Psychology: Human Perception \& Performance, 15, 816-825.

Gillam, B., \& Grant, T., Jr. (1984). Aggregation and unit formation in the perception of moving collinear lines. Perception, 3, 659-664.

GoGEL, W. (1974). Relative motion and the adjacency principle. Quarterly Journal of Experimental Psychology, 26, 425-437.

Johansson, G. (1950). Configurations in event perception. Uppsala: Almqvist \& Wiksell.

Johansson, G. (1964). Perception of motion and changing form. Scandinavian Journal of Psychology, 5, 181-208.

Johansson, G. (1973). Visual perception of biological motion and a model for its analysis. Perception \& Psychophysics, 14, 201-211. 
KOENDERINK, J. J. (1986). Optic flow. Vision Research, 26, 161-180. KoENDERINK, J. J., \& VAN DOORN, A. J. (1975). Invariant properties of the motion parallax field due to movement of rigid bodies relative to an observer. Optica Acta, 22, 773-791.

Lappin, J. S., Norman, J. F., \& Mowafy, L. (1991). The detectability of geometric structure in rapidly changing optical patterns. Perception, 20, 513-528.

Wallach, H., Becklen, R., \& NitzberG, D. (1985). Vector analysis and process combination in motion perception. Journal of Experimental Psychology: Human Perception and Performance, 11, 93-102.

WERTHEIMER, M. (1923). Untersuchungen zur Lehre von der Gestalt. Psychologische Forschung, 4, 301-350.

(Manuscript received November 25, 1991; revision accepted for publication June 26,1992 .) 